\title{
Artículos
}

\section{Estigmatización territorial y salud: experiencias de desigualdad social en la periferia de Buenos Aires}

\author{
Territorial stigmatization and health: Experiences \\ of social inequality in the periphery of Buenos Aires
}

\author{
Betina Freidin ${ }^{\mathrm{a}}$ \\ Matías S. Ballesteros ${ }^{b}$ \\ Mercedes Krause ${ }^{c}$ \\ Agustín Wilner $^{d}$
}

\section{Resumen}

Abordamos las experiencias de estigmatización territorial y sus efectos en la salud de las mujeres y sus familias en un barrio popular del conurbano de Buenos Aires, Argentina. Los datos provienen de grupos focalizados realizados durante 2015 y 2016, y entrevistas a referentes barriales. Reconstruimos procesos macro y mesoso-

${ }^{a}$ Universidad de Buenos Aires, Facultad de Ciencias Sociales, Instituto de Investigaciones Gino Germani, y Consejo Nacional de Investigaciones Científicas y Técnicas (Conicet). Dirección: Uriburu 950, piso 6, 1114, Ciudad Autónoma de Buenos Aires, Argentina. Correo: frei dinbetina@gmail.com ORCID: http://orcid.org/0000-0001-7458-2160

${ }^{b}$ Universidad de Buenos Aires, Facultad de Ciencias Sociales, Instituto de Investigaciones Gino Germani, y Consejo Nacional de Investigaciones Científicas y Técnicas (Conicet), Argentina. Correo: matiballesteros@yahoo.com.ar ORCID: https://orcid.org/0000-0003-1321-2777

${ }^{\mathrm{c}}$ Universidad de Buenos Aires, Facultad de Ciencias Sociales, Instituto de Investigaciones Gino Germani, y Consejo Nacional de Investigaciones Científicas y Técnicas (Conicet), Argentina. Correo: merkrause@gmail.com ORCID: https://orcid.org/0000-0002-3724-6413

${ }^{d}$ Universidad de Buenos Aires, Facultad de Ciencias Sociales, Instituto de Investigaciones Gino Germani, y Consejo Nacional de Investigaciones Científicas y Técnicas (Conicet), Argentina. Correo: aguswilner@gmail.com ORCID: https://orcid.org/0000-0003-0006-2124

Nota de los autores: Este trabajo se deriva de un proyecto de investigación más amplio sobre el cuidado de la salud, financiado por la Secretaría de Ciencia y Técnica de la Universidad de Buenos Aires: "Desigualdad social, cultura y salud: recursos y prácticas cotidianas frente al imperativo del cuidado" (Proyecto UBACyT 20020130100594, 2014-2017). Agradecemos a todas las personas que colaboraron con nuestro estudio por su buena disposición e interés y por el tiempo que nos dedicaron, muy especialmente a las mujeres que participaron en los grupos focalizados, a los referentes barriales y al equipo de salud del Centro de Atención Primaria del barrio. Los evaluadores anónimos nos aportaron constructivos comentarios. 
ciales de discriminación estructural que producen desventajas territoriales, vulnerabilidades y riesgos para la salud de los habitantes, y contribuyen a la estigmatización espacial. Analizamos las responsabilidades por las condiciones deterioradas de vida atribuidas a los vecinos y a actores extrabarriales. Asimismo, damos cuenta de algunas estrategias individuales y colectivas para afrontar la estigmatización territorial.

Palabras clave: estigmatización territorial; salud; discriminación estructural; Buenos Aires.

\begin{abstract}
We address the experiences of territorial stigmatization and their impact on health among a group of women who live in a working class-neighborhood of the periphery of Buenos Aires, Argentina. Data come from focus groups conducted during 2015 and 2016 and from interviews with neighborhood representatives. We reconstruct macro and meso-social processes of structural discrimination that produce placebased disadvantages, vulnerabilities, risks, and that also contribute to the neighborhood stigmatization. We analyze the responsibilities allocated to the neighbors and external actors. In addition, we account for some individual and collective strategies to face the territorial stigmatization.
\end{abstract}

Keywords: territorial stigmatization; health; structural discrimination; Buenos Aires.

\title{
Introducción
}

El lugar donde las personas viven afecta su salud. Diversos estudios epidemiológicos que abordan la temática en distintas escalas -regional, nacional y en el interior de los países- documentan la relación entre la cercanía a fuentes de contaminación ambiental con la incidencia de enfermedades infecciosas y crónicas no transmisibles, la salud mental, las expectativas de vida saludable y la mortalidad (Tunstall, Shaw y Dorling, 2004; Evans y Kantrowitz, 2002; WHO, 2002).

Desde hace varias décadas, en Latinoamérica un conjunto de estudios empíricos ha abordado la relación entre el hábitat y la salud, focalizando en las condiciones de vida de los sectores populares. Para el caso argentino, Cuenya, Almada, Armus, Castells, Di Loreto y Peñaiva (1985) estudiaron la problemática en un asentamiento informal en el conurbano de Buenos Aires, dando cuenta del acceso a los servicios de salud, especialmente en el primer nivel de atención, y las patologías prevalecientes entre niños y adultos. Di Virgilio (2003) analizó la condición de salud y las estrategias de cuidado, considerando la heterogeneidad interna socioocupacional y del hábitat de las 
familias de un barrio pobre de la ciudad de Buenos Aires. En una línea similar destacan los estudios realizados en otros contextos nacionales, entre ellos, los de Martínez Salgado (1997), Rivera Márquez (1997) y Castillejos y Serrano (1997) en colonias pobres de la Ciudad de México, y la investigación de Fadda y Jirón (2001) en barriadas chilenas. Otros trabajos se han centrado más específicamente en los procesos de segregación socioterritorial y su impacto en la salud ambiental en barrios con alta degradación del Área Metropolitana de Buenos Aires, por su cercanía a actividades industriales contaminantes y a rellenos sanitarios (Auyero y Swistun, 2008; Curutchet, Grinberg y Gutiérrez, 2012; Grinberg, Dafunchio y Mantiñán, 2013; entre otros). Más recientemente, se han incluido en el análisis los efectos de la estigmatización de los territorios urbanos relegados sobre la salud de los habitantes y sus respuestas ante el estigma que pesa sobre el lugar en el que viven. Esta línea de trabajo está en desarrollo (Graham, Padilla, Lopez, Stern, Peterson y Keene, 2016) y los estudios empíricos corresponden mayormente a las áreas periféricas de las metrópolis de países centrales (Pearce, 2012). En Argentina, el trabajo de Kessler (2012) es pionero en esta dirección; su estudio en un complejo habitacional del conurbano bonaerense aborda cómo la estigmatización afecta negativamente a sus moradores y agrava privaciones ya existentes para los grupos en situación de pobreza, siendo la salud una de las múltiples dimensiones analizadas por el autor.

El propósito del presente artículo es contribuir a la literatura empírica sobre la salud y la estigmatización territorial, a partir de una investigación que realizamos con mujeres de un barrio de clase popular del conurbano norte de Buenos Aires. El trabajo de campo se extendió desde el año 2015 hasta principios de 2018. Nos planteamos como objetivos: reconstruir procesos macro y mesosociales de discriminación estructural que producen desventajas territoriales, vulnerabilidades y riesgos para la salud de los habitantes del barrio, y contribuyen a la estigmatización espacial; analizar las responsabilidades atribuidas a los vecinos y a los actores extrabarriales por las condiciones deterioradas de vida; y dar cuenta de algunas estrategias individuales y colectivas para afrontar la estigmatización territorial.

El artículo tiene la siguiente estructura. Primero presentamos teóricamente la problemática más general sobre salud, desigualdad social y territorio urbano, integrando debates de la sociología médica, la epidemiología social crítica, la salud colectiva latinoamericana y los estudios de la salud de la población (population health). Segundo, desde teorizaciones provenientes de la sociología urbana y ambiental y de la geografía humana, desarrollamos el concepto de estigmatización territorial para dar cuenta de la experiencia subjetiva y colectiva de la segregación socioterritorial y su impacto en la 
salud psicofísica, sintetizando asimismo algunos trabajos empíricos internacionales y locales representativos de esta línea de investigación. Tercero, describimos las características del barrio donde llevamos a cabo nuestra investigación y los aspectos metodológicos. Cuarto, presentamos los resultados del estudio, y quinto, las conclusiones.

\section{Desigualdad social, territorio y salud}

Desde la epidemiología social crítica y la salud colectiva latinoamericana, se ha puesto el foco en las condiciones objetivas que inciden en las desigualdades en la calidad de vida de los grupos sociales, la cual está en estrecha relación con los procesos de salud - enfermedad - atención - cuidado (PSEAC), así como en la vulnerabilidad diferencial socioterritorial en el espacio urbano frente a la degradación, depredación y polución ambiental que genera la economía capitalista globalizada (Breilh, 2010). Siguiendo a Blanco Gil y López Orellano (2007, p. 114), el concepto de territorio refiere a la articulación específica del conjunto de soportes materiales de los procesos de reproducción de los diferentes grupos sociales, incluyendo el equipamiento urbano, el acceso a los servicios de salud y transporte, el tipo y calidad de la vivienda, el medio geofísico humanizado, y las características culturales y sociodemográficas de la población. Así se identifican territorios-población vulnerabilizados en términos de riesgos para la salud y acceso a la infraestructura sanitaria (Blanco Gil y López Orellano, 2007, p. 114). Se asume la historicidad de la salud de los grupos poblacionales y el carácter dinámico del espacio urbano, con sus múltiples y complejas determinaciones sociales (macro-micro), que operan de manera jerárquica y con contradicciones (Breihl, 2010). La importancia de analizar la salud a escala barrial -e intrabarrial- radica en que es en el territorio donde se desarrollan modos de vida colectivos derivados de las relaciones de clase en entrecruzamiento con las étnicas y de género. Los modos de vida colectivos resultantes pueden ser protectores o destructivos de la salud (Breihl, 2010, pp. 88-89). Asimismo, en el nivel comunitario se pueden desarrollar capacidades y soportes colectivos para el empoderamiento y mejoría de la calidad de vida de los grupos subalternos. Los estilos de vida-condicionados por los modos de vida- se constituyen a lo largo del tiempo a través de prácticas familiares e individuales como formas de vivir que resultan protectoras o perjudiciales, forjando capacidades individuales positivas para el desarrollo personal, o bien vulnerabilidades psicofísicas (Breihl, 2010, p. 90). 
El estudio de los contextos locales en relación con las desigualdades sociales en la salud ha sido privilegiado en los últimos años por el enfoque de la salud de la población (population health). Esta línea de trabajos de corte cuantitativo pone el foco en los denominados "efectos del barrio" -y en áreas geográficas más pequeñas-relativos a aspectos ambientales que inciden en la salud de sus habitantes, distinguiendo el ambiente físico del social. El ambiente físico incluye no sólo la exposición a riesgos ambientales "tradicionales" (entre ellos, contaminación del aire, acceso a redes de agua segura y saneamiento, exposición a residuos tóxicos industriales), sino también a aspectos construidos relativos al diseño urbano y los espacios públicos, y al acceso a lugares de provisión de alimentos saludables y oportunidades recreativas. Mientras que el ambiente social refiere a las relaciones interpersonales, los niveles de seguridad y violencia, y otros aspectos de la organización social local (Diez Roux y Mair, 2010). Estos elementos afectan la salud de múltiples formas, pues limitan o facilitan los comportamientos de los sujetos -así como generan estrés o lo mitigan- con respecto al apoyo social y a las relaciones interpersonales (Diez Roux y Mair, 2010, p. 128).

Por su parte, los estudios sociológicos sobre las desigualdades en la salud señalan que la composición étnica y social de las distintas áreas, así como los aspectos socioculturales e históricos, impactan en la salud mediante las acciones de ayuda mutua y otras formas de capital social y recursos colectivos para el cuidado (Carpiano, Link y Phelan, 2008; Annandale, 2015). Son las comunidades económicamente más desfavorecidas las que sufren mayor carga de inequidad ambiental y cuentan con menores recursos colectivos, como parques y espacios abiertos recreativos, para incrementar la calidad de vida (Auyero y Swistun, 2008; Ross, 2011; Evans y Kantrowitz, 2002). Para la población urbana, el derecho a la salud ambiental se ubica en el marco más amplio del derecho a la ciudad, tanto en el nivel colectivo como en el individual (Ugalde, 2015). La desigualdad socioambiental incluye el mayor riesgo de victimización por episodios de violencia interpersonal en los barrios vulnerabilizados de la periferia urbana, lo que afecta la salud física, emocional y mental de sus habitantes (Kilanski y Auyero, 2015; Evans y Kantrowitz, 2002). Así es que, desde una perspectiva de justicia ambiental, los grupos marginalizados sufren una doble inequidad: la individual por su desventaja estructural, y la derivada de su localización en los peores ambientes físicos y sociales (Frohlich y Abel, 2014). En este sentido, Diez Roux y Mair (2010, p. 131) concluyen que la segregación territorial y la desigualdad en el acceso a los recursos se retroalimentan: la segregación residencial puede resultar en desigualdades espaciales en recursos, lo que a su vez puede reforzar la segregación residencial. Se enfatiza, en esta línea, el efecto 
acumulativo y potencialmente sinergético de los múltiples factores ambientales y estresores sociales que afectan la salud de los grupos sociales más vulnerables (Morello-Frosch, Zuk, Jerrett, Shamasunder y Kyle, 2011).

En la sociología médica también ha adquirido centralidad el concepto de lugar ( place) para analizar las desigualdades en salud. Mientras el espacio alude a la localización geofísica, el lugar describe lo que significa esa localización, llevando la atención a las interrelaciones entre las personas y los espacios que habitan o transitan, es decir, al plano de la experiencia vivida que influye sobre las acciones individuales y colectivas vinculadas con la salud (Annandale, 2015, p. 99; Popay, Thomas, Williams, Bennett, Gatrell, y Bostock, 2003, p. 56). Se señala la importancia de analizar cómo se construyen estas interacciones y la forma en la que distintos grupos conciben las desigualdades territoriales en términos de "buenos" y "malos" lugares para vivir, teniendo en cuenta las características del área, pero también los comportamientos de sus residentes. Asimismo, la relación entre privación material, riesgos y comportamientos relacionados con la salud está influenciada por la capacidad de los sujetos de construir una identidad personal positiva pese a la desventaja territorial, que incluye procesos de categorización para diferenciarse de "otros" a los que les atribuyen responsabilidades por los problemas que los afectan (Annandale, 2015; Popay et al., 2003).

\section{Estigmatización territorial y salud}

Desde la sociología urbana y ambiental y la geografía humana, se remarca la importancia de la estigmatización territorial para dar cuenta de la experiencia subjetiva y colectiva de la segregación socioterritorial y sus efectos en la salud psicofísica (Pearce, 2012). El concepto de estigmatización territorial fue desarrollado por Wacquant con el propósito de incluir el lugar en el proceso social, político e institucional de creación del estigma sobre la base del descrédito, retomando la teoría del poder simbólico de Bourdieu y la conceptualización de Goffman sobre el manejo de las identidades dañadas (spoiled identities) (Slater, 2015). Si bien el concepto de estigma ha recibido distintas definiciones y usos en la psicología social para abordar el proceso cognitivo de creación de categorías y estereotipos, los enfoques sociológicos sobre cómo opera el estigma territorial parten de la elaboración de Goffman acerca de los atributos desacreditantes y la discriminación implicada que se construyen de manera relacional y dinámica, y en condiciones de asimetría de poder (Link y Phelan, 2001; Kessler, 2012). En este sentido, Wacquant, Slater y Pereira, (2014, p. 1270) especifican que "la estigmatización territo- 
rial no es una condición estática o un proceso neutral, sino una forma injuriosa de acción con consecuencias, que opera a partir de representaciones colectivas sobre un lugar". El estigma territorial asocia a los residentes de determinadas áreas con rasgos negativos, como el peligro, la criminalidad y la suciedad, que se desplazan de los individuos a los grupos, y finalmente a los espacios mismos (Kornberg, 2016, p. 264). Se busca avanzar en la comprensión teórica y empírica de cómo esta forma de discriminación se produce, difunde y opera por parte de agencias burocráticas y comerciales, así como en la vida diaria (Wacquant, Slater y Pereira, 2014). Se abordan las estructuras simbólicas en la producción de desigualdades y marginalidad en áreas relegadas del espacio urbano producidas desde "arriba" (discursos científicos, del periodismo y de los funcionarios públicos, etc.) y desde "abajo" (las representaciones que circulan en las conversaciones y en los encuentros cotidianos) para avanzar en el conocimiento de este proceso social, así como también para reorientar políticas sociales e implementar formas efectivas de activismo comunitario (Slater, 2015; Wacquant, Slater y Pereira, 2014).

Un interrogante que orienta los estudios empíricos es observar de qué modo la estigmatización territorial genera privaciones específicas para las poblaciones afectadas, y cómo agrava las ya existentes (Kessler, 2012). Los casos abordados en los países desarrollados corresponden a barrios urbanos periféricos construidos en los discursos públicos como lugares a los que hay que evitar ir ("no-go zones"), representación que perjudica a las localizaciones ya estigmatizadas por sus altos niveles de pobreza, delitos, segregación racial, y que también experimentaron abandono de inversión pública y privada (Pearce, 2012, p. 1926; Graham et al., 2016; Kornberg, 2016). En este sentido, Slater (2015, p. 5) advierte que constituye un desafío analítico separar (disentangle) los efectos relativamente autónomos de la estigmatización territorial de otras numerosas formas en las que los residentes de barrios de clase baja son descalificados. Siguiendo a Link y Phelan (2001), Kessler (2012, p. 173) remarca que a lo largo del tiempo el estigma genera "efectos acumulativos que repercuten en las condiciones de vida más allá de la presencia visible de un agente estigmatizador en un momento dado, lo que han llamado discriminación estructural". El concepto remite a un proceso de decisiones discriminatorias acumulativas de agentes ubicados en distintos niveles de responsabilidad en instituciones públicas y privadas, incluyendo la desinversión local, "sin que haya quedado labrado en ningún lado que tal exclusión estuvo vinculada al estigma" (Kessler, 2012, p. 180). Pertenecer a una comunidad estigmatizada puede también resultar en una menor legitimidad para demandar inversión municipal para mejorar el barrio, y la deci- 
sión de inversión en un lugar difamado puede significar un menor rédito político para los funcionarios (Kessler, 2012, p. 180).

Las respuestas de los residentes de las áreas afectadas ante el estigma son diversas. A escala comunitaria, la internalización de la estigmatización territorial puede socavar la solidaridad de clase y la acción colectiva; o bien, resistir la difamación puede tener un efecto de autoafirmación y orgullo por el lugar (Slater, 2015; Kessler, 2012). Mientras que, en el ámbito individual, Wacquant, Slater y Pereira (2014) señalan que las personas pueden desarrollar distintas estrategias para afrontar el estigma territorial: aceptarlo y reproducirlo -incluyendo prácticas de microdiferenciación y de denigración "lateral"-, o bien, resistir la categorización impuesta; de estas respuestas dependen las distintas trayectorias en el espacio físico y social.

Desde el campo de las desigualdades sociales en salud, Slater (2015, pp. 12-13) sostiene que la creciente literatura epidemiológica sobre los "efectos del barrio" se ha centrado en dinámicas que operan en sus límites territoriales, descuidando los efectos de la mirada negativa de los otros sobre éste y sus habitantes. Siguiendo a Wacquant, Slater y Pereira (2014), Pearce (2012, p. 1923) remarca que los barrios son construcciones políticas e históricas que representan procesos sociales y económicos de distinta escala que se acumulan a lo largo del tiempo. Este autor propone un modelo conceptual de varios caminos institucionalizados e individualizados, que no son mutuamente excluyentes, para explicar cómo el estigma espacial puede impactar negativamente en la salud (Pearce, 2012, pp. 1927-1929). Primero, la internalización del estigma afecta la identidad personal y las relaciones sociales, y ambos elementos están relacionados con las prácticas de salud y el bienestar psicoemocional. Además, la etiqueta negativa es móvil, es decir, los habitantes pueden "transportarla" con ellos a otros lugares, mediando ésta las interacciones cotidianas y en diversas instituciones (los servicios de salud, por ejemplo). Segundo, el estigma territorial que denota "inferioridad moral" también crea desventajas en las oportunidades de vida a lo largo del tiempo, en particular, en las educacionales y laborales, que están relacionadas causalmente con la salud. Tercero, la construcción social de los espacios juega un papel vital en los patrones de inversión y desinversión estatal y privada que afectan las oportunidades disponibles para sus residentes, incluyendo la vivienda, las obras de infraestructura y los servicios. Asimismo, las relaciones interpersonales pueden verse perjudicadas por el retraimiento hacia la esfera privada, afectando la salud psicofísica. Como consecuencia de estos procesos interrelacionados, Pearce (2012, p. 1924) concluye que la estigmatización territorial puede afectar negativamente la salud de múltiples maneras, que incluyen las identidades individuales y las relaciones sociales, los 
determinantes sociales de la salud, así como las respuestas institucionales para tratar problemas sociales muy arraigados.

Considerado un campo de indagación en emergencia (Graham et al., 2016), los trabajos empíricos sobre salud y estigma territorial se han llevado a cabo en países centrales posindustrializados, en relación con los procesos de segregación social y racial de las poblaciones marginalizadas de los barrios urbanos. Estudios como los de Graham et al. (2016) y Thomas (2016), analizan las consecuencias de la estigmatización del territorio en las relaciones sociales, la construcción de identidades y la consideración de los otros. El estudio de Tabuchi et al. (citado en Pearce, 2012) realizado en Japón, muestra que las experiencias de discriminación geográfica se asocian con una mayor probabilidad de presentar signos depresivos y diagnósticos de salud mental. En Argentina, el trabajo de Kessler (2012) aborda las consecuencias de la estigmatización territorial de los habitantes de un complejo habitacional del conurbano bonaerense. Aunque no está centrado en temas de salud, el estudio analizó cómo la estigmatización producida especialmente por el discurso mediático opera sobre sus moradores y las personas que entablan relaciones con ellos, produciendo o profundizando situaciones previas de exclusión o privación social (Kessler, 2012, p. 168). Las privaciones incluían la recolección irregular de la basura y el acceso limitado al transporte público, a remises [autos de alquiler] que no eran del barrio, así como a ambulancias. Auyero y Berti (2013, pp. 39-41) estudiaron la multiplicidad de violencias que viven cotidianamente los habitantes de un barrio marginalizado de la periferia de Buenos Aires. La investigación también documenta "la dimensión material del abandono estatal" por la falta de obras de infraestructura y servicios, que expone a los vecinos a riesgos para la salud y al "sufrimiento ambiental". Auyero y Swistun (2008) desarrollan este concepto en un estudio previo, focalizándose en un barrio con gran exposición a la contaminación industrial por su proximidad a un polo petroquímico. Si bien el trabajo no se centra en la dimensión simbólica de la estigmatización territorial, la descripción del barrio encuadra en los "lugares a donde no ir", especialmente en relación con la inseguridad urbana, además de los riesgos ambientales y las condiciones de vida insalubres en la que se encuentran sus residentes (Auyero y Swistun, 2008, p. 45).

\section{Características del barrio donde se realizó la investigación}

El barrio seleccionado está localizado en el norte del segundo cordón del conurbano bonaerense, en una zona donde conviven urbanizaciones cerradas 
con diversas modalidades de hábitat popular. Al este, está circundado por el río Reconquista (el segundo más contaminado del país), y al sur el límite es un cerco de alambre que rodea un muro levantado por un barrio cerrado. A su vez, de este a oeste, el barrio está atravesado por un canal que desemboca en el río mencionado, que fue parcialmente entubado hace unos años.

El barrio presenta una importante heterogeneidad socioterritorial. La información cuantitativa sobre las condiciones materiales de vida y el perfil socioeconómico del barrio en su totalidad es escasa y parcial, y proviene de encuestas con muestras probabilísticas realizadas en 2013 y 2014 por el Centro de Atención Primaria, que lo tiene bajo su área programática. En el barrio se distinguen distintas áreas según el tipo de viviendas (la mayoría con paredes y pisos de material, pero algunas de ellas son precarias, tipo casilla) y el trazado urbano (pocas calles aún no están asfaltadas ni señalizadas, y pequeñas áreas no cuentan con trazado de manzanas ni numeración postal de las viviendas). Algunos terrenos bajos son inundables, especialmente los cercanos al río Reconquista y los linderos a la urbanización cerrada. El relevamiento realizado en el sector más relegado del barrio permite caracterizar a sus residentes en cuanto a su educación formal, inserción en el mercado de trabajo, condición migratoria y situación habitacional, y comparar estos indicadores con los del conjunto de los partidos del Gran Buenos Aires, según el Censo Nacional de Hogares, Población y Viviendas del año 2010. ${ }^{1}$

En el sector más relegado del barrio, un tercio de las personas encuestadas residen en viviendas con hacinamiento crítico y cerca del $10 \%$ de las viviendas son casillas, ranchos o cuartos en inquilinatos, frente al 7.5 y $3.9 \%$, respectivamente, del conjunto del Gran Buenos Aires. Con relación al perfil educativo, entre las personas de 25 años y más de esta zona del barrio, alrededor del 20\% no finalizó el nivel primario, y menos del 25\% terminó los estudios secundarios, mientras que en el conjunto de los partidos del Gran Buenos Aires esos porcentajes corresponden al 12.4 y $40.9 \%$, respectivamente. La tasa de desocupación superaba para el año 2013 el 10\%, y el porcentaje de trabajadores en relación de dependencia y cuentapropistas que no realizaban aportes jubilatorios casi alcanzaba a la mitad, mientras que en el Gran Buenos Aires, en 2010, esos porcentajes correspondían al 6.9 y $36.1 \%$, respectivamente. Por su parte, el porcentaje de extranjeros supera el $20 \%$, de los cuales aproximadamente tres cuartos son paraguayos, mientras que en el conjunto del Gran Buenos Aires los extranjeros constituyen el 7.6\% y

1 El partido en el que se encuentra el barrio, considerado en su totalidad, tiene mejores resultados en estos indicadores que el promedio de los partidos del Gran Buenos Aires.

Estudios Demográficos y Urbanos, vol. 35, núm. 1 (103), enero-abril, 2020, pp. 153-183 
los paraguayos el $45 \%$ de ellos. Sintetizando, el perfil educativo y las condiciones habitacionales y laborales de los residentes de esta zona son peores que los del conjunto de los partidos del Gran Buenos Aires, y es más alto el porcentaje de extranjeros. Cabe destacar además que a ninguna zona del barrio llega la red de cloacas (carencia que afecta a casi la totalidad de viviendas del partido), mientras que la red de gas natural no alcanza al área del barrio donde realizamos el estudio, pero sí es un servicio del que disponen los residentes de la "mejor" área; esto es, cruzando el zanjón semientubado hacia el lado norte.

Como lo señalamos en la sección precedente, los barrios emblemáticos de la estigmatización territorial son aquellos que adquieren notoriedad negativa en los discursos públicos y mediáticos, como lo son villas, asentamientos y barrios de viviendas sociales ubicados en los lugares más bajos de la jerarquía del espacio urbano. El barrio en que nosotros realizamos el estudio no corresponde a dicha caracterización, al permanecer más anónimo en las representaciones mediáticas y el conocimiento popular. No obstante, sus habitantes afrontan cotidianamente algunas de las consecuencias de la discriminación espacial. De hecho, la estigmatización de la "peor zona" del barrio fue reconocida por las autoridades municipales en el discurso de inauguración de una obra de infraestructura años atrás, el entubamiento parcial de un canal afluente del río Reconquista, que opera como una frontera geográfica y simbólica al dividir las calidades de vida y las identidades barriales de uno y otro lado. El discurso fue reproducido en medios periodísticos locales e hizo explícita la discriminación estructural que afectaba al área más postergada, al anunciar que la obra pública significaría el fin de las divisiones sociales para los vecinos, además de disminuir el riesgo ambiental y la inseguridad. Cuando años después nosotros realizamos el trabajo de campo, las desventajas territoriales persistían. Por otra parte, utilizando medios sociales -como una página de Facebook sobre las noticias del barrio-, grupos de vecinos hicieron denuncias de hechos delictivos y monitorearon la denominada "zona roja". Aludieron con este adjetivo a unas pocas cuadras donde se concentraban los delincuentes y a partir de lo cual el barrio es "famoso" por aspectos negativos, aunque esta difamación opere localmente. A diferencia de los trabajos realizados en barrios emblemáticos de la estigmatización territorial construidos como "lugares a los que no hay que ir" en discursos que circulan "desde arriba y desde lejos en tonos sombríos y monocromáticos" (Wacquant, 2007, p. 1, citado en Auyero y Swistun, 2008, p. 45), nuestro estudio aborda procesos de estigmatización territorial que operan en distintas escalas, incluyendo el nivel más local de segregación y microdiferenciación que afecta la vida cotidiana y la salud de sus habitantes. 


\section{Metodología}

Los datos cualitativos que analizamos en este artículo provienen de seis grupos focalizados con mujeres del barrio durante los años 2015 y 2016, en el marco de un proyecto de investigación más amplio sobre el cuidado de la salud. ${ }^{2}$ Participaron 39 mujeres de entre 18 y 60 años, en grupos compuestos por entre cinco y diez participantes. Para realizar el trabajo de campo nos contactamos inicialmente con el centro de salud (Centro de Atención Primaria de Salud, CAPS). Tras varias reuniones para interiorizarnos en la problemática sociosanitaria del barrio y del trabajo del CAPS y de las postas de salud [establecimientos sanitarios de primer nivel, de baja complejidad] ubicadas en las zonas de mayor vulnerabilidad, la dirección y los médicos residentes que colaboraron con nuestro estudio nos contactaron con promotoras de salud y referentes de organizaciones barriales (un comedor comunitario, un bachillerato popular, postas, talleres de oficios y un centro de estimulación temprana, nucleados por una ONG religiosa). El CAPS tiene una larga trayectoria de trabajo en conjunto con estas organizaciones y participa en la red comunitaria que las aglutina. Los referentes contactados nos facilitaron ingresar a dichas organizaciones para realizar allí los grupos focalizados.

La mayoría de las participantes de los grupos vivían en la parte considera como la "peor", ${ }^{3}$ correspondiente al lado sur delimitado por el zanjón principal y el río Reconquista. La presencia de unas pocas participantes que vivían del "mejor" lado del zanjón y en un barrio de una localidad lindera brindó cierta diversidad interna, lo que nos permitió observar problemáticas socioambientales propias de la comunidad seleccionada, así como otras que eran comunes a las localidades cercanas.

Los grupos focalizados abordan conversaciones colectivas alrededor de un tema, guiadas por un moderador o moderadora (Morgan y Krueger, 1993), cuya tarea consiste en orientar la conversación para favorecer el intercambio de puntos de vista y experiencias entre los participantes. Mediante el "efecto del grupo" se busca alcanzar una comprensión más rica de la que podría lograrse con entrevistas personales (Johnson, 1996; Freidin, 2016). La guía de pautas para conducir los grupos fue semiestructurada. La moderación estuvo a cargo de la directora de la investigación. Los integrantes del equipo tomaron notas de la dinámica grupal y de los contenidos de las conversacio-

2 "Desigualdad social, cultura y salud: recursos y prácticas cotidianas frente al imperativo del cuidado", Universidad de Buenos Aires.

3 Seguimos las distinciones entre uno y otro lado del zanjón realizadas por las mujeres que participaron en los grupos, y utilizamos los adjetivos por ellas utilizados para caracterizarlos. 
nes, e intervinieron retomando temas y planteando preguntas. Las discusiones grupales tuvieron una duración aproximada de dos horas, y fueron grabadas digitalmente y transcritas verbatim.

Las experiencias vinculadas con la estigmatización territorial emergieron explícitamente en los grupos. De manera complementaria, indagamos sobre la temática en entrevistas con referentes barriales, realizadas durante los años 2016, 2017 y 2018. Para analizar las experiencias de estigmatización territorial, reconstruimos procesos macro y mesosociales referidos a la discriminación estructural por parte del Estado, que, por acción u omisión, generan desventajas territoriales, vulnerabilidades y riesgos diferenciales para la salud de los habitantes del "peor" lado y contribuyen a su estigmatización, observando la retroalimentación de estos procesos. Asimismo, analizamos la asignación de responsabilidades, vinculadas con las condiciones deterioradas de vida, a algunos vecinos del barrio, así como a actores extrabarriales. Finalmente, analizamos cómo la estigmatización afecta la identidad barrial e individual, y estudiamos qué mecanismos de diferenciación interna se ponen en marcha, así como también algunos de los soportes colectivos desarrollados localmente para mejorar la calidad de vida en el barrio.

Utilizamos el programa de análisis de datos cualitativos Atlas.ti para codificar las transcripciones. Para resguardar el anonimato de las participantes, cambiamos los nombres y eliminamos las referencias a personas, organizaciones, establecimientos, calles y barrios, que pudieran permitir su identificación (de igual modo, anonimizamos los testimonios de los referentes barriales). Este procedimiento se garantizó en la carta de consentimiento informado que entregamos a todos los participantes del estudio.

\section{Dos barrios en uno: las consecuencias de la discriminación estructural}

Las diferencias entre uno y otro lado del zanjón semientubado son notables, en términos de infraestructura pública, acceso a servicios y calidad de vida. Como lo sintetizó una participante, "ya cuando cruzás el zanjón, ya ves la diferencia; no hace falta que nadie te cuente la diferencia que hay, porque vos la ves cuando cruzás el zanjón" (Lila, Grupo 4). A continuación, describimos las privaciones específicas que operan en el barrio que vulneran el derecho a la ciudad, que afectan la salud psicofísica y ambiental de su población, y que contribuyen a su estigmatización territorial.

La principal evidencia de la discriminación estructural que sufre el barrio es la obra inconclusa del canal principal que lo atraviesa y que demar- 
ca "dos barrios diferentes en uno", como lo expresó una de las participantes del Grupo 4. La primera etapa de esta obra contempló el entubamiento de parte del canal, el relleno de la cuenca y la construcción de un boulevard sobre el que se emplazarían espacios verdes, incluyendo un circuito aeróbico, bicisendas y juegos para niños al lado del CAPS, luminarias y la construcción de una escuela secundaria. Fue anunciada públicamente por el funcionario político que la inauguró como punto final al "estigma" que pesaba sobre el lado peor del barrio, además de como una intervención para disminuir el riesgo ambiental y la inseguridad. Sin embargo, la obra de relleno y entubamiento no prosiguió y el edificio escolar sin terminar permanece abandonado. El zanjón continúa siendo una fuente de riesgos de accidentes y es un lugar donde se acumula basura. También opera como una frontera simbólica que limita la interacción y la sociabilidad cotidiana entre los vecinos de uno y otro lado. Pero, sobre todo, visibiliza la elusión gubernamental en la realización de obras de infraestructura y de trabajos de saneamiento periódico, lo cual contribuye al deterioro de la salud ambiental y al aislamiento. El zanjón que divide el barrio y mantiene a un segmento de su población "apartada" en la peor zona es una evidencia de los mecanismos institucionales que perpetúan las desventajas territoriales y que contribuyen a la estigmatización espacial de los barrios populares del conurbano bonaerense.

Las calles del "mejor" lado del zanjón se encuentran asfaltadas en su totalidad, cuentan con veredas [aceras] elevadas y anchas, separadas de la calle por cordones de material. En cambio, pese a los reclamos de los vecinos del "peor" lado, algunas calles continúan sin asfaltar y las obras de pavimentación avanzan lentamente. Las calles y las veredas se encuentran a la misma altura y muchas veces están atravesadas por zanjas pequeñas que no están tapadas, las cuales dificultan la circulación peatonal (y de quienes utilizan sillas de ruedas) y automotriz (incluidas las ambulancias), por lo que aumentan los riesgos de accidentes y la contaminación. Además, dicha parte del barrio no cuenta con la señalización de varias calles ni la numeración de algunas de las viviendas. Así, el servicio de correo no llega a algunos domicilios en la nueva zona de viviendas sociales, y se dificulta la ubicación de algunas de éstas por parte de las personas externas al barrio. Ante una emergencia médica, el problemático acceso vehicular puede significar la diferencia entre la vida y la muerte, como en el caso de una vecina que murió de un edema de glotis porque "la ambulancia no sabía cómo ingresar al barrio", lo que nos contó una de las referentes barriales.

Se suma a estas desventajas que el tendido de la red de gas natural sí cubre la totalidad del lado "mejor" del barrio, pero sólo parcialmente al "peor", lo que los obliga a usar garrafas, con el consecuente gasto para las 
familias y riesgos para la salud. El servicio de recolección diferenciada de residuos reciclables, que es parte de la política nacional y provincial de desarrollo de municipios saludables y sustentables, sólo cubre al lado "mejor" del barrio.

En cuanto a la integración del lado "peor" del barrio al resto de la trama urbana, éste se encuentra relativamente aislado, ya que la única línea de colectivos que llega hasta el fondo recorre el boulevard con paradas del lado "mejor"; otras líneas de colectivo pasan por la ruta, casi en el límite norte. Ello implica que los peatones deben cruzar el zanjón por alguna de sus pasarelas, las cuales fueron señaladas por las participantes como uno de los puntos donde frecuentemente ocurren episodios de inseguridad. Para salir en remís [auto de alquiler] o en vehículo particular, también deben circular por el otro lado del barrio hasta llegar a la ruta, ya que el límite este del barrio hacia la autopista se encuentra cercado.

Las desventajas territoriales no se agotan en las hasta aquí descritas. La oferta comercial del "mejor" lado es más amplia, diversificada y concentrada sobre una avenida principal. En relación con los pequeños comercios de alimentos, algunas mujeres en el Grupo 4 plantearon sus dudas sobre la calidad y la seguridad alimentaria de los productos a la venta del lado "peor", apuntando especialmente a las condiciones de refrigeración de los alimentos frescos y a los posibles riesgos de contaminación cruzada. Aunque no todas las participantes estuvieron de acuerdo con dicha caracterización, esta circunstancia conlleva riesgos directos para la salud, además de implicar un mayor esfuerzo en los desplazamientos cotidianos para abastecerse de alimentos saludables, lo cual puede afectar las prácticas alimentarias de los hogares (Diez Roux y Mair, 2010; Evans y Kantrowitz, 2002). La segregación perpetuada por la desinversión estatal para mejorar el barrio también desalienta emprendimientos comerciales privados, lo que contribuye a la estigmatización del área (Slater, 2015; Pearce, 2012).

El lado "peor" también cuenta con menos espacios públicos para actividades físicas y recreativas. Para realizarlas, los vecinos tienen que trasladarse al boulevard que se construyó sobre el zanjón y donde se trazó un circuito aeróbico, a las plazas que se encuentran del lado "mejor", o al polideportivo municipal al que se accede cruzando el zanjón, atravesando la otra parte del barrio y cruzando la ruta por un puente peatonal. Las escuelas y jardines de infantes que las mujeres prefieren para sus hijos también están del "otro" lado. Las diferencias entre uno y otro lado del zanjón, más la falta de conectividad en términos de la red de transporte público y acceso directo por vías de conexión interurbana, llevan a que la circulación diaria de los vecinos sea unidireccional. Las participantes transitan la "mejor" 
parte del barrio cotidianamente para satisfacer distintas necesidades, mientras que la circulación por la "peor" parte del barrio se restringe a sus propios habitantes, salvo excepciones como las visitas a familiares:

Mara: La gente de allá no cruza para acá. Nosotros para hacer las compras, para salir a la ruta, para tomar un colectivo, para todo tenemos que ir para allá. Ellos para acá no tienen nada que hacer.

Mirta: ¿Ni siquiera para las instituciones, ponele, para la capilla, para un jardín, no?

Mara: Y van a las capillas de aquel lado. Y para el jardín tampoco porque con esto también... del radio...

Mirta: Porque es por el radio, claro.

Mara: La gente de aquel barrio no va a venir a los jardines acá [Grupo 4].

"Ellos para acá no tienen nada que hacer", fue una expresión empleada en los grupos que condensa la realidad cotidiana del flujo unidireccional de personas en el barrio, y el sentimiento de relegación y valoración negativa del lugar compartido por varias de las mujeres.

Otro aspecto en el que puede observarse cómo opera la discriminación estructural sobre el barrio está vinculado con los emprendimientos inmobiliarios que se construyeron desde los años noventa en el partido. La elevación de terrenos de las urbanizaciones cerradas que fueron rodeando partes del barrio implicó una nueva desventaja territorial frente al riesgo de inundación. Mara, en el Grupo 4, cuenta que su casa no se inundaba antes de la construcción de un barrio cerrado lindero a su propiedad debido a que su terreno se encuentra en una zona alta. Sin embargo, actualmente se inunda cuando llueve fuerte debido a que el emprendimiento privado abre compuertas que vuelcan el agua excedente hacia el barrio. A ello se suma que se construyó un paredón que está circundado por una zanja para que drene el exceso de agua de lluvia, pero debido a la falta de limpieza y saneamiento por parte del municipio, del lado donde viven las participantes se llenó de pastizales y de basura, impidiendo que cumpla la función de drenaje. El pastizal abandonado contribuye además a la mala imagen del barrio porque se ha constituido en un foco de inseguridad. Los contrastes entre uno y otro lado del muro que describen las participantes reflejan los cambios en la periferia urbana de Buenos Aires, que siguiendo a Svampa (2001), acompañaron las transformaciones de la estructura social y la lógica global de privatización del espacio urbano iniciado en los setenta (véase también Di Virgilio, 2015). La consecuente segregación espacial dio como resultado "la incrustación de nichos de riqueza en extendidos bolsones de pobreza" (Svampa, 2001, p. 86) en los que se disfruta de un ambiente verde y bucólico, del cual se priva a 
los barrios empobrecidos. Sin embargo, no todas las participantes asignan responsabilidad a los barrios cerrados por el agravamiento de la problemática de las inundaciones. Algunas sostienen que la responsabilidad la tienen los vecinos del barrio por la basura que arrojan en el zanjón próximo al paredón, y la municipalidad por la falta de limpieza. Estas distintas interpretaciones tienen implicaciones en los reclamos que los vecinos pueden hacer a la delegación municipal con el fin de que intervenga sobre los distintos problemas que confluyen en el deterioro de la calidad de vida en el barrio y en su imagen externa. Dificultan asimismo la identificación de la ausencia estatal en la prevención de las "externalidades" negativas de las nuevas urbanizaciones sobre las condiciones de vida en los barrios de los pobres.

En suma, el acceso diferencial a la infraestructura urbana y los servicios visibiliza la falta de prioridad en la asignación de recursos públicos y en las políticas de urbanización, lo que a su vez contribuye a la desinversión de los agentes privados. Estos procesos de largo plazo perjudican la calidad de vida de los vecinos del lado "peor" y redundan en la estigmatización del barrio.

\section{La higiene del barrio: las actitudes de los vecinos y el "mal aspecto" para los de afuera}

La falta de higiene barrial preocupa a las mujeres por los riesgos para la salud que conlleva la presencia cotidiana de basura, pero también por el estigma territorial que genera. Para la mayoría de las participantes, son algunos de los vecinos los responsables del "mal aspecto" del barrio. El papel del servicio municipal de recolección y el control del Estado para promover y mejorar la salud ambiental en el barrio fueron considerados en las conversaciones grupales, pero la mayor responsabilidad fue atribuida a los propios vecinos.

Mara, en el Grupo 4, describe el problema de la falta de higiene en distintos espacios públicos concluyendo que, "lamentablemente vivimos, por lo menos de este lado del barrio, en la mugre, literalmente". En el arroyo parcialmente entubado que atraviesa el barrio se puede encontrar todo tipo de desechos, incluyendo "una heladera vieja, un sillón, un colchón", cuenta Isabel en el Grupo 5. Los chicos, por su parte, utilizan las bolsas de basura de la calle como arcos de fútbol frente a una de las escuelas, y los perros callejeros hacen su parte rompiendo las bolsas y desparramando la basura. Se suma la basura acumulada en el pequeño zanjón y en el pastizal lindero al muro de la urbanización cerrada que, como señalamos, se encuentra abandonado por el servicio de recolección municipal. Y fundamentalmen- 
te preocupa la basura acumulada en "el terraplén", un gran pastizal que abarca unas dos manzanas linderas a una posta de salud ubicada en la zona más relegada del barrio, que con el tiempo se transformó en un basural clandestino a cielo abierto.

La preocupación por los perjuicios de la basura para la salud fue recurrente en las conversaciones grupales en relación con la trasmisión de enfermedades infecciosas, pero también por el daño para la salud mental que genera vivir en esas condiciones, lo que está en línea con los estudios epidemiológicos internacionales. Se suman a los riesgos el "mal aspecto" del barrio que la acumulación de basura ofrece para "toda la gente que viene de otro lado":

Susana: Si hay basura es por la gente. Porque el basurero pasa todos los días, el de las ramas pasa martes y sábado o martes y jueves, y el que tira basura en el zanjón es porque es sucio.

Teresa: Tienen el mal hábito.

Susana: Porque pasa el basurero. Si no tenés basurero, un coso [cosa] para poner la basura, colgalo en el árbol, en un palo, pero ya porque la gente alguna es sucia. Vos ves en el zanjón bolsas, lavarropas... ¿Por qué tiran en el zanjón, que da mala vista al barrio? Si vos tenés una heladera vieja, ponela ahí en tu terreno ahí afuera, que pasa el camión y la lleva. Una heladera vieja, un sillón, un colchón. ¿Por qué tienen que ir a tirar allá en el zanjón?, que toda la gente que viene de otro lado, qué sé yo, es mal aspecto del barrio, no sé, si pasan los basureros.

Gisela: Y viste que hay gente que barre la calle también.

Susana: Sí, todos...

Gisela: Todos los días barren, pero para la tarde ya está todo una mugre [Grupo 5].

En este fragmento vemos cómo las participantes responsabilizan a algunos vecinos por la suciedad del barrio, y consideran que el municipio cumple correctamente con la función de recolección y limpieza. Este argumento primó entre las participantes de los grupos que se diferenciaron de los vecinos "crotos", "sucios" y "mugrientos". Una vecina del Grupo 5 sintetiza las demarcaciones simbólicas entre los vecinos respecto de la responsabilidad que les cabe por la suciedad del barrio y sus implicaciones para la construcción identitaria de unos y otros, señalando que "todos no somos iguales":

Porque te da rabia, porque vos tenés todo limpito en tu casa, cortás el pasto, no dejás un papelito, yo ando alzando los huesos de los perros, que quede lindo. Ya el vecino me ve, corta el pasto también, y vos salís a media cuadra y están los pañales tirados [en la calle], todo [...] Todos no somos iguales [Susana, Grupo 5]. 
La distinción moral que realizan con los "malos" vecinos puede entenderse, siguiendo a Wacquant, Slater y Pereira (2014), como una estrategia de microdiferenciación para afrontar y resistir el estigma territorial que afecta a todos los habitantes por igual ante la mirada externa. En la medida que esta estrategia es emprendida desde el plano individual, desplaza el estigma hacia otros habitantes del barrio (aquellos que por su conducta serían "merecedores" del mismo), por lo que constituye una forma de interiorizar el estigma y de reproducirlo.

Algunas participantes adjudicaron este y otros comportamientos, como las quemas de basura que contaminan el aire y dan una mala imagen colectiva, a los grupos más vulnerables del barrio. Fundamentalmente a los jóvenes, "los pendejos que andan en la calle. Tienen frío y queman" -afirma Patricia en el Grupo 2- y a los "carreros" que viven de la recolección de cartones y materiales reciclables para su venta utilizando carros desplazados por caballos. Estas demarcaciones simbólicas operan en el barrio, aunque se reconozca el efecto perverso de los procesos de categorización negativa de los que se encuentran en condiciones de máxima privación material y relegación social, "los pobres discriminamos a los más pobres", por cómo viven y por el impacto negativo que tiene en el barrio:

Esa es la parte más triste donde la gente que vive en esa zona, los pobres discriminamos a los más pobres porque esa gente sigue viviendo en ranchos que tienen techo de lona. Ni hablar de agua, de higiene, la basura es parte de la vivienda, el caballo, el carro, todo es parte de una misma cosa [Mara, Grupo 4].

Siguiendo a Grinberg, Dafunchio y Mantiñán (2013, p. 127), queda claro que la forma de percibir el entorno y de relacionarse con la basura en la vida cotidiana varía en función de si se encuentra en ella un medio de vida o subsistencia. Lo que para algunos constituye una fuente de contaminación y un desperdicio, para otros tiene valor de uso o de cambio. Así es que otros vecinos también muy vulnerabilizados en sus condiciones de vida recogen desechos, que los carreros depositan en el terraplén para venderlos (como objetos con cobre) o para usarlos (maderas para calefaccionar el hogar y cocinar).

Si bien la relación que algunos de los vecinos con condiciones de vida más precarizadas tienen con la basura puede explicar parte de la problemática del basural, operan otros factores estructurales vinculados con la falta de provisión de servicios públicos y controles municipales, así como con el accionar de personas ajenas al barrio. Vecinas y referentes consultados coincidieron en que comerciantes de la zona depositan diariamente desechos en 
el terraplén. Si bien hay un servicio de recolección de residuos en el área, el mismo no alcanza a retirar toda la basura acumulada y no hay ningún tipo de control municipal para erradicar el basural. Por otra parte, cuando realizamos el trabajo de campo, el basurero y el camión recolector no pasaban por algunas calles que permanecían cerradas porque la obra de viviendas sociales había quedado interrumpida. Si bien en esa zona hay contenedores puestos por el municipio, algunas mujeres aseguraron que, según la ubicación de su vivienda, tenían que caminar varias cuadras para utilizarlos, y que, por miedo a que las roben durante la caminata, quemaban la basura o la dejaban directamente en la vereda.

Quemar o no basura es una práctica que varias de las participantes consideran que distingue a los que les interesa y a los que son indiferentes a la higiene barrial, por sus efectos contaminantes. Teresa, por ejemplo, al contar cómo era la situación en el área más próxima a su vivienda, aclaró que ni ella ni sus vecinos de la cuadra quemaban basura, aunque tuvieran que caminar un trecho buscando algún cesto donde dejar las bolsas. Su testimonio, junto con los de otras pocas participantes y referentes barriales, deja en evidencia que al menos en determinadas cuadras el servicio de recolección no funciona eficientemente. Esta deficiencia contribuye a la degradación ambiental y a las quemas. Podemos observar cómo una conjunción de problemáticas y carencias en los servicios municipales contribuye a generar comportamientos que luego son atribuidos a malas actitudes individuales de los vecinos, las que a su vez son fuentes de distinciones morales entre unos y otros. La quema de basura se atribuye a las "malas costumbres". Sin embargo, esta práctica adquiere un sentido particular en un contexto donde la opción de no quemarla es caminar varias cuadras consideradas inseguras hasta llegar a un cesto o contenedor. Las deficiencias del Estado para garantizar la seguridad y el servicio de recolección de basura en determinadas áreas promueven las quemas, por las que luego los vecinos son estigmatizados internamente, constituyendo además una práctica que se suma a la valoración negativa del barrio en su conjunto.

\section{Segregación socioterritorial, violencia interpersonal e inseguridad}

Otra de las formas en la que se vive la estigmatización territorial es la segregación a causa de la violencia interpersonal e inseguridad que, según los testimonios reunidos, se concentra en la parte "peor" del barrio. La expresión "nos tienen apartadas" (Grupo 5) da cuenta de experiencias de aislamiento, como cuando los remises que no son del lugar no quieren entrar al área por 
su "mala fama". Los recorridos suelen llegar hasta el zanjón principal, que actúa como una frontera socioterritorial:

Gisela: Te dicen: “¿Del otro lado del zanjón? No, no entramos”. Alcanzamos todos, los que vivimos acá y los que viven para allá, y por ahí... [se superponen: "tienen miedo"] [...] Es como que nos tienen apartadas. Yo cuando vengo en remís a la noche de [menciona el centro de la localidad], me dejan en [...] vivo en la otra cuadra, me deja en el parque este [en el boulevard]. No, porque no cruzan para acá. Antes era un arroyo, ahora viste que se entubaron algunas partes y están todas entubadas. Pero nos tienen como que las de allá "mejorcitas", y que las de acá somos "las peores" [...].

Moderadora: ¿Ustedes también sienten esto que dice Gisela?

Gisela: Todos lo sufrimos.

Silvia: Sí, es verdad eso.

Gisela: Todos lo sufrimos. Yo hace veinticuatro años que vivo acá. Nunca, gracias a Dios nunca me pasó nada, pero toda la gente...

Teresa: A veces, según, algunos [remises], hay algunos que entran, algunos, no todos $[\ldots]$.

Susana: No, si yo vengo de [menciona otro barrio], le digo "del otro lado del arroyo a media cuadra". Me dice: "No, la dejo de este lado".

Moderadora: $\mathrm{Y}$ si tienen que llevar a algún familiar y no tienen auto, ¿cómo hacen?

Teresa: Yo mayormente [cuando no estoy en el barrio] llamo a los remiseros de acá $[\ldots]$ Me van a buscar y vuelven.

Moderadora: El tema es venir de afuera.

Teresa: Exactamente.

Gisela: Sí, porque tiene mala fama el lado este [Grupo 5].

La violencia simbólica vivida por Gisela es generalizada; las divisiones entre el "peor" y el "mejor" lado del barrio se trasladan a sus habitantes y separan a las mujeres como "mejorcitas" y "peores". Una jerarquización que deriva del lugar en la que cada cual vive y que tiene consecuencias directas para la salud. Al igual que muchos de los remises que no son del barrio, las ambulancias difícilmente entran en determinadas zonas. Como nos cuenta una de las referentes entrevistadas, "llamás a la ambulancia y te dice que a esa parte no quiere entrar". La resistencia para ingresar a la "peor" zona sin custodia policial se relaciona con robos y episodios de violencia que han sufrido las ambulancias en el pasado, como un apedreo tras la muerte de un niño, que los vecinos atribuyeron a la demora en llegar.

Pero no sólo hay lugares del barrio construidos desde afuera como lugares a los que es mejor no ir; también los propios habitantes demarcan territorios que prefieren evitar y que en relación a la salud implica el desa- 
provechamiento de recursos sanitarios y de espacios públicos. Teresa (Grupo 5) prefiere atenderse en el CAPS que ir a una posta ubicada a cinco cuadras de su vivienda, aunque tenga que caminar más de veinte cuadras o ir en bicicleta. La razón es el "mal ambiente" asociado con la violencia callejera, la acumulación de basura, el consumo de drogas y los robos. La sensación de vulnerabilidad también lleva a algunas mujeres a no caminar solas por el barrio cuando terminan su jornada laboral; otras lo hacen solamente de día y acompañadas por sus maridos. Sólo una de las participantes del Grupo 1 aseguró que podía caminar sola por la plaza sin problemas, incluso de noche.

Los robos sucesivos y el vandalismo deterioran las postas. Si bien la "mala fama" pesa mayormente sobre la mitad del barrio que queda al sur del zanjón, en él se distingue la "zona roja" donde se concentrarían los delincuentes. Las postas sanitarias cercanas han sufrido robos de equipamiento y daños en las instalaciones en varias ocasiones. Según una de las participantes del Grupo 3, que fue testigo del hecho por vivir frente a una de las postas, los atacantes tuvieron tiempo para volver al lugar y seguir robando por la tardanza de la policía en arribar. Si bien el municipio había asignado personal de seguridad en las postas, debido a los recortes presupuestarios el servicio se reemplazó por una alarma que no funcionaba, situación que implicó mayor desprotección y exposición a sufrir daños. Para preservar una de las postas, como nos comentaron las referentes, los mismos vecinos comenzaron a cercarla con un muro de ladrillos que levantaban los fines de semana.

El clima de violencia interpersonal en la calle y la inseguridad llevan a prácticas de vigilancia familiar sobre los niños y adolescentes. Algunas participantes refieren el encierro cuando describen los cuidados y restricciones que imponen a sus hijos por la presencia de "juntas" en las esquinas. Mara (Grupo 4) no deja que su hija de 10 años salga sola a la calle bajo ninguna circunstancia, aunque la niña "muere por ir a ver qué pasa del otro lado del portón, porque las ve a las nenas en la calle, a los nenes en la calle todo el día; de ahí a la esquina hay dos pasos"; y para que vaya a la escuela que queda a 25 cuadras de su casa paga un transporte privado, con el esfuerzo económico que representa el gasto para su familia. A diferencia de la otra localidad cercana donde viven Mirta y Lila, que participaron del mismo grupo y relataron prácticas de vigilancia similares, lo distintivo del territorio estigmatizado parece ser que las malas "juntas" no sólo son vistas como posibles atacantes, sino también como potenciales amistades. Lo expresa crudamente una de las participantes del Grupo 1, quien tras la muerte de su hija se hizo cargo de su nieto de 16 años, "hay pocas amistades buenas en el barrio, así que hay que estar [con él]". 
La vigilancia familiar y la sociabilidad restringida de los menores son respuestas ante la falta de protección estatal frente a los problemas de violencia interpersonal e inseguridad. Al mismo tiempo, contribuyen a reproducir la estigmatización territorial por diferenciaciones de lugares y personas del "peor" lado basadas en categorizaciones negativas como el "mal ambiente", la "mala fama", las "juntas" y las "malas amistades". Siguiendo a Graham et al. (2016), el aislamiento respecto de los residentes estigmatizados es una forma de internalizar el estigma y una estrategia de preservación personal.

\section{Respuestas colectivas frente al estigma territorial}

La responsabilidad por la basura constituye una fuente de conflicto entre los vecinos que conduce a microestrategias de denigración lateral, para evitar que el estigma territorial que la basura genera pese sobre todos los vecinos, en lugar de afectar sólo a quienes lo merecerían. Esta dinámica de construcción identitaria individual se refuerza con el poco éxito que tuvieron algunas incitativas propuestas desde el CAPS, en conjunto con organizaciones barriales, para generar una mayor conciencia colectiva e intervenciones para la promoción de la salud ambiental (talleres y charlas, prácticas de reciclado de botellas, jardinería y jornadas de limpieza de algunas cuadras). La falta de participación refuerza la distinción entre los vecinos, como lo expresa Inés en el Grupo 4: "La gente que interviene por lo general no es la gente que hace el desastre [...] todos vemos las cosas de diferente manera. Entonces conseguimos hacer en conjunto pocas cosas". Clara, en el mismo grupo, cuenta que en la zona de las viviendas sociales los recién llegados no pueden hacer nada frente a las actitudes de los viejos residentes porque se generan situaciones de violencia: "Para ellos es normal y si vos les decís algo, estás en problemas porque vienen, te apedrean la casa. Es gente que no podés dialogar". La falta de participación y de posibilidades de diálogo refuerza las divisiones morales entre "nosotros" y "ellos" y socava la solidaridad para coordinar acciones colectivas.

Los episodios de violencia interpersonal e inseguridad perpetuadas entre los vecinos, comunes a otras áreas periféricas del conurbano bonaerense (Auyero y Berti, 2013), generan la vivencia de una vulnerabilidad compartida (Alarcón, 2016). Frente a ella, también se desarrollan estrategias colectivas de protección. Por ejemplo, en el Grupo 3 nos contaron que los hombres de la comunidad paraguaya se unen para "correr" a las "juntas" y dispersarlas. Si bien estas acciones expresan solidaridad intragrupal, también tienen el efecto de estigmatizar a los niños y jóvenes más vulnerabilizados 
que se encuentran en una situación de fuerte desprotección social y familiar, además de reforzar divisiones étnicas. Pero, por otro lado, para estos niños y jóvenes se han desarrollado soportes comunitarios desde el CAPS y otras organizaciones para abordar problemáticas individuales y comunitarias. Una de las respuestas que reciben de algunos jóvenes es que ellos no viven en un barrio cerrado, lo que naturaliza las diferencias en las calidades de vida entre los que más y menos tienen. Aun así, han logrado pequeños cambios que resultan protectores tanto de los jóvenes como del barrio en su conjunto. Se trata de estrategias de resistencia colectiva frente a la estigmatización territorial. Otras iniciativas también dan visibilidad a las formas de resistencia a aceptar vivir en un lugar que sufre de discriminación estructural, lo que contribuye a su difamación. Reclamos hechos a la municipalidad para tapar las zanjas en las veredas, contar con más iluminación y extender el servicio de recolección de basura para evitar las quemas, se orientan en esta dirección. Siguiendo a Carman y Janoschka (2015), estas demandas expresan resistencias frente a las políticas públicas de abandono y son formas de ejercer ciudadanía urbana.

Retomando a Kessler (2012), vemos que la relación entre la estigmatización territorial, los lazos sociales y la acción colectiva es contingente. Si por un lado vivir en un área difamada resiente los vínculos interpersonales y exacerba las microdiferenciaciones, por el otro, sienta las bases para emprender acciones colectivas más allá de los resultados que se obtengan a lo largo del tiempo.

\section{Conclusiones}

A partir de los relatos de las mujeres que participaron en los grupos focalizados y de los testimonios de las referentes barriales, podemos concluir que el estigma territorial potencia la vulnerabilidad de este territorio-población. Si la segregación socioterritorial y las desigualdades socioeconómicas se retroalimentan (Diez Roux y Mair, 2010), podemos considerar al estigma territorial como un mediador de esta relación, dado que condiciona el acceso a distintos recursos. El estigma territorial potencia la acumulación de desventajas y los procesos de discriminación estructural, al tiempo que afecta las identidades colectivas e individuales de los habitantes del barrio. Incorporar el estigma como una dimensión del concepto "lugar" nos permite profundizar y complejizar el análisis de las desigualdades sociales en la salud en relación con la exposición a riesgos socioambientales y al acceso a recursos sanitarios, y dar visibilidad a cómo se viven subjetivamente las privaciones. 
Los modos en los que el estigma territorial se reproduce cotidianamente muestran cómo procesos de estigmatización que operan "desde arriba" y "desde abajo" se articulan en escalas intrabarriales. Observamos mecanismos continuos de producción del estigma territorial entre ambos polos, ya que, por ejemplo, los mismos vecinos del barrio demarcan zonas "que hay que evitar", cuyas imágenes circulan en los medios sociales. Construyen jerarquías morales con los vecinos que descuidan la higiene barrial porque sus conductas los exponen a riesgos para la salud y contribuyen a la "mala imagen" del barrio, la que a su vez redunda en una mayor marginación y aislamiento de los que viven en las zonas más relegadas estructuralmente. Internamente, las distinciones morales entre los vecinos contribuyen a erosionar las bases de solidaridad para realizar acciones colectivas, y también redundan en una menor capacidad de demanda ante el municipio. No obstante, algunas iniciativas dan visibilidad a formas de resistencia a aceptar vivir en un lugar que sufre de discriminación estructural, lo que contribuye a su difamación. Reclamos hechos a la municipalidad para tapar zanjas en las veredas, contar con más iluminación y extender el servicio de recolección de basura para evitar las quemas, se orientan en esta dirección. Estas demandas expresan resistencias frente a las políticas públicas de abandono, y pueden verse como formas de reclamo del derecho a la ciudad (Carman y Janoschka, 2015). Asimismo, tratándose de un barrio con una alta organización territorial, se han desarrollado soportes comunitarios para los grupos altamente estigmatizados internamente (los niños y jóvenes más vulnerabilizados que integran las "juntas", que contribuyen con sus conductas en los espacios públicos a la mala imagen barrial). Se trata de prácticas de empoderamiento comunitario (Breihl, 2010) que buscan mejorar la calidad de vida de los sectores subalternos en territorios relegados del espacio urbano, aunque no sean los más emblemáticos de la estigmatización territorial.

\section{Bibliografía}

Alarcón, C. (2016). Entrevista: Relatos periodísticos sobre lo real-real. En B. Focás y O. Rincón (comps.), (In)seguridad, medios y miedos: una mirada desde las experiencias y las prácticas cotidianas en América Latina (pp. 11-16). Cali, Colombia: Universidad Icesi, Facultad de Derecho y Ciencias Sociales.

Annandale, E. (2015). The sociology of health and medicine. Cambridge: Polity. 
Auyero, J. y Berti, M. F. (2013). La violencia en los márgenes. Buenos Aires, Argentina: Katz.

Auyero, J. y Swistun, D. (2008). Inflamable: estudio del sufrimiento ambiental. Buenos Aires, Argentina: Paidós.

Blanco Gil, J. y López Orellano, O. (2007). Condiciones de vida, salud y territorio. Un campo temático en (re)construcción. En E. C. Jarrillo Soto y E. Guinsberg (coords.), Temas y desafios de salud colectiva (pp. 103125). Buenos Aires, Argentina: Lugar.

Breilh, J. (2010). La epidemiología crítica: una nueva forma de mirar la salud en el espacio urbano. Salud Colectiva, 6(1), 83-101. Recuperado de http://www.redalyc.org/articulo.oa?id=73115246007

Carman, M. y Janoschka, M. (2015). Ciudades en disputa: estudios urbanos críticos sobre conflictos y resistencias. QUID 16, Revista del Área de Estudios Urbanos Instituto Gino Germani, 4, 1-7. Recuperado de http:// contested-cities.net/blog/ciudades-en-disputa-estudios-urbanos-criticossobre-conflictos-y-resistencias/

Carpiano, R., Link, B. y Phelan, J. (2008). Social inequality and health: Future directions for the fundamental cause explanation. En A. Lareau y D. Conley (eds.), Social class: How does it work? Nueva York, NY: Rusell Sage Foundation.

Castillejos, M. y Serrano, P. (1997). Efectos de los contaminantes atmosféricos sobre la salud en cuatro colonias. En M. Schteingart (coord.), Pobreza, condiciones de vida y salud en la Ciudad de México (pp. 629692). Ciudad de México: El Colegio de México, A.C.

Cuenya, B., Almada, H., Armus, D., Castells, J., Di Loreto, M. y Peñaiva, S. (1985). Condiciones de hábitat y salud de los sectores populares: un estudio piloto en el asentamiento San Marín de Quilmes (Informes de Investigación del CEUR, núm.1). Buenos Aires, Argentina: Centro de Estudios Urbanos y Regionales.

Curutchet, G., Grinberg, S. y Gutiérrez, R. A. (2012). Degradación ambiental y periferia urbana: un estudio transdisciplinario sobre la contaminación en la región metropolitana de Buenos Aires. Ambiente y Sociedade, 15(2): 173-194. Recuperado de http://www.scielo.br/pdf/asoc/v15n2/10. pdf

Di Virgilo, M. (2003). Hábitat y salud: estrategias de las familias pobres. Buenos Aires, Argentina: Lumiere.

Di Virgilio, M. (2015). Urbanizaciones de origen informal en Buenos Aires. Lógicas de producción de suelo urbano y acceso a la vivienda. Estudios Demográficos y Urbanos, 30(3), 651-690. Recuperado de https://estu diosdemograficosyurbanos.colmex.mx/index.php/edu/article/view/1496 
Diez Roux, A. V. y Mair, C. (2010). Neighborhoods and health. Annals of the New York Academy of Sciences, 1186(1), 125-145. Recuperado de https://nyaspubs.onlinelibrary.wiley.com/doi/full/10.1111/j.17496632.2009.05333.x

Evans, G. W. y Kantrowitz, E. (2002). Socioeconomic status and health: The potential role of environmental risk exposure. Annual Review of Public Health, 23, 303-331. Recuperado de https:/www.annualreviews.org/ doi/abs/10.1146/annurev.publhealth.23.112001.112349

Fadda, J. y Jirón, P. (2001). Calidad de vida y género en sectores populares urbanos. Un estudio de caso en Santiago de Chile: síntesis final y conclusiones. Revista INVI, 16(42), 105-138. Recuperado de https://www. redalyc.org/articulo.oa?id $=25804207$

Freidin, B. (2016). Revisando el uso de los grupos focalizados en la investigación social. Revista Latinoamericana de Metodología de las Ciencias Sociales, 6(1), 1-28. Recuperado de http://sedici.unlp.edu.ar/handle/ $10915 / 54509$

Freidin, B. (2017). Introducción. En B. Freidin (coord.), Cuidar la salud: mandatos culturales y prácticas cotidianas de la clase media en Buenos Aires (pp. 11-31). Buenos Aires, Argentina: Imago Mundi.

Frohlich, K. y Abel, T. (2014). Environmental justice and health practices: Understanding how health inequities arise at the local level. En S. Cohn (ed.), From health behaviours to health practices: Critical perspectives (pp. 43-56). Cardiff, Gales: Willey Blackwell.

Graham, L. F., Padilla, M., Lopez, W., Stern, A., Peterson, J. y Keene, D. (2016). Spatial stigma and health in postindustrial Detroit. International Quarterly of Community Health Education, 36(2), 105-113. Recuperado de https://journals.sagepub.com/doi/pdf/10.1177/0272684X15627800

Grinberg, S., Dafunchio, S. y Mantiñán, L. M. (2013). Biopolítica y ambiente en cuestión: lugares de la basura. Horizontes Sociológicos, 1(1), 115-142. Recuperado de https://ri.conicet.gov.ar/bitstream/handle/11336/15711/Conicet_Digital_Nro.19037.pdf?sequence=2\&is Allowed $=\mathrm{y}$

Johnson, A. (1996). It's good to talk. The focus group and the sociological imagination. The Sociological Review, 44(3), 517-538. Recuperado de https://onlinelibrary.wiley.com/doi/abs/10.1111/j.1467-954X.1996. tb00435.x

Kessler, G. (2012). Las consecuencias de la estigmatización territorial. Reflexiones a partir de un caso particular. Espacios en blanco. Serie Indagaciones, 22(1), 165-198. Recuperado de http://www.scielo.org.ar/pdf/ eb/v22n1/v22n1a07.pdf 
Kilanski, K. y Auyero, J. (2015). Introduction. En J. Auyero, P. Burgois y N. Scheper-Hughes (eds.), Violence at the urban margins (pp. 1-17). Oxford: Oxford University Press.

Kornberg, D. (2016). The structural origins of territorial stigma: Water and racial politics in metropolitan Detroit, 1950s-2010s. International Journal of Urban and Regional Research, 40(2), 263-283. Recuperado de https://onlinelibrary.wiley.com/doi/full/10.1111/1468-2427.12343

Link, B. y Phelan, J. (2001). Conceptualizing stigma. Annual Review of Sociology, 27, 363-385. Recuperado de https://www.jstor.org/stable/ 2678626?seq=1\#metadata_info_tab_contents

Martínez Salgado, C. (1997). Aspectos metodológicos para el estudio de la morbilidad por encuesta en comunidades pobres urbanas. En M. Schteingart (coord.), Pobreza, condiciones de vida y salud en la Ciudad de México (pp. 525-537). Ciudad de México: El Colegio de México. A.C.

Morello-Frosch, R., Zuk, M., Jerrett, M., Shamasunder, B. y Kyle, A. (2011). Understanding the cumulative impacts of inequalities in environmental health: Implications for policy. Health Affairs, 30(5), 879-887. Recuperado de https://www.healthaffairs.org/doi/pdf/10.1377/hlthaff.2011.0153

Morgan, D. L. y Krueger, R. A. (1993). When to use focus groups and why? En D. L. Morgan (comp.), Successful focus groups. Advancing the state of the art (pp. 3-19). Newbery Park, CA: Sage Focus Edition.

Pearce, J. (2012). The blemish of place: Stigma, geography and health inequalities. A commentary on Tabuchi, Fukuhara \& Iso. Social Science and Medicine, 75(11), 1921-1924. Recuperado de https://www.science direct.com/science/article/pii/S0277953612005874

Popay, J., Thomas, C., Williams, G., Bennett, S., Gatrell, A. y Bostock, L. (2003). A proper place to live: Health inequalities, agency and the normative dimensions of space. Social Science and Medicine, 57(1), 55-69. Recuperado de https://www.sciencedirect.com/science/article/pii/ S027795360200299X

Rivera Márquez, J. A. (1997). Condiciones de salud-enfermedad en las colonias populares. En M. Schteingart (coord.), Pobreza, condiciones de vida y salud en la Ciudad de México (pp. 539-628). Ciudad de México: El Colegio de México, A.C.

Ross, C. E. (2011). Collective threat, trust, and the sense of personal control. Journal of Health and Social Behavior, 52(3), 287-296. Recuperado de https://doi.org/10.1177/0022146511404558

Slater, T. (2015). Territorial stigmatization: Symbolic defamation and contemporary metropolis. En J. Hannigan y G. Richards (eds.), The Sage handbook of new urban studies (pp. 111-125). Londres: Sage. 
Svampa, M. (2001). Los que ganaron: la vida en los countries y barrios privados. Buenos Aires, Argentina: Biblos.

Thomas, G. (2016). It's not that bad: Stigma, health, and place in a postindustrial community. Health and Place, 38, 1-7. Recuperado de https:// www.sciencedirect.com/science/article/pii/S1353829216000034

Tunstall, H., Shaw, M. y Dorling, D. (2004). Places and health. Journals Epidemiology and Community Health, 58, 6-10. Recuperado de https:// jech.bmj.com/content/jech/58/1/6.full.pdf

Ugalde, V. (2015). Derecho a la ciudad, derechos en la ciudad. Estudios Demográficos y Urbanos, 30(3), 567-595. Recuperado de https://estu diosdemograficosyurbanos.colmex.mx/index.php/edu/article/view/ $1494 / 1487$

Wacquant, L., Slater, T. y Pereira, V. (2014). Territorial stigmatization in action. Environment and Planning A: Economy and Space, 46(6), 12701280. Recuperado de https://journals.sagepub.com/doi/pdf/10.1068/ a4606ge

WHO. (2002). The World Health Organization 2002 Report: Reducing risks, promoting healthy life. Ginebra: WHO. Recuperado de https://www. who.int/whr/2002/en/summary_riskfactors_chp4.pdf

\section{Acerca de los autores}

Betina Freidin es socióloga y maestra en Investigación Social por la Universidad de Buenos Aires (UBA), y doctora en Sociología por Brandeis University, Estados Unidos. Es investigadora independiente del Conicet, con sede en el Instituto de Investigaciones Gino Germani, UBA. También es profesora adjunta de "Metodología y técnicas de investigación social" en la carrera de Sociología de la UBA. Sus principales temáticas de estudio son: las desigualdades sociales en salud, las prácticas de cuidado de la salud, el campo profesional de las medicinas alternativas y la metodología cualitativa. ORCID: http://orcid.org/0000-0001-7458-2160

Entre sus publicaciones se encuentran:

Freidin, B., Wilner, A., Ballesteros. M. S. y Krause, M. (2018). Salud, riesgo ambiental y territorio: las experiencias de mujeres que viven en un barrio de clase popular en la periferia de Buenos Aires. Buenos Aires: Teseo.

Freidin, B. (coord.) (2017). Cuidar la salud: mandatos culturales y prácticas cotidianas de la clase media en Buenos Aires. Buenos Aires: Imago Mundi. 
Freidin, B. (2014). Proyectos profesionales alternativos: relatos biográficos de médicos que practican medicinas no convencionales. Buenos Aires: Imago Mundi.

Matías S. Ballesteros es sociólogo, maestro en Investigación Social y doctor en Ciencias Sociales por la Universidad de Buenos Aires (UBA). Es investigador asistente del Conicet, con sede en el Instituto de Investigaciones Gino Germani de la UBA, institución en la que también es integrante del equipo de investigación dirigido por la doctora Betina Freidin. Es docente de "Metodología y técnicas de investigación social" en la carrera de Sociología de la UBA. Dirige la investigación "Desigualdades sociales en estilos y modos de vida vinculados con la salud en zonas urbanas de Argentina, 2007-2014", enmarcado en el Proyecto de Reconocimiento Institucional de Investigaciones de la Facultad de Ciencias Sociales de la misma universidad. Sus principales temáticas de estudio son: la desigualdad social, la sociología de la salud y la metodología cuantitativa. ORCID: https://orcid.org/00000003-1321-2777

Entre sus publicaciones se encuentran:

Ballesteros, M. S. (2016) Profesionales de la salud en el primer nivel de atención en Argentina: un análisis sobre las desigualdades jurisdiccionales. Revista Geograficando, 12(2).

Ballesteros, M. S. (2016). Desigualdades sociales en los tiempos de espera para la consulta médica en Argentina. Revista Gerencia y Políticas de Salud, 15(30), 234-250.

Ballesteros, M. S. y Freidin, B. (2015). Reflexiones sobre la conceptualización y la medición del acceso a los servicios de salud en Argentina: el caso de la Encuesta Nacional de Factores de Riesgo 2009. Salud Colectiva, 11(4), 523-535.

Mercedes Krause es doctora en Ciencias Sociales, maestra en Investigación en Ciencias Sociales y socióloga por la Facultad de Ciencias Sociales de la Universidad de Buenos Aires (UBA). Es docente de licenciatura y posgrado de la materia "Metodologías y técnicas de la investigación" e investigadora del Instituto de Investigaciones Gino Germani de la UBA; es integrante de los equipos de investigación dirigidos por las doctoras Ruth Sautu y Betina Freidin en el área de Estratificación Social. Sus temas de interés son: las metodologías de investigación, la fenomenología social, las desigualdades sociales en salud, el análisis de clases sociales y las teorías de la interseccionalidad. ORCID: https://orcid.org/0000-0002-3724-6413

Entre sus publicaciones se encuentran: 
Freidin, B., Wilner, A., Ballesteros. M. S. y Krause, M. (2018). Salud, riesgo ambiental y territorio: las experiencias de mujeres que viven en un barrio de clase popular en la periferia de Buenos Aires. Buenos Aires: Teseo. Krause, M. y Ballesteros, M. S. (2018). Interseccionalidad en desigualdades en salud en Argentina: discusiones teórico-metodológicas a partir de una encuesta poblacional. Hacia la Promoción de la Salud, 23(2), 13-33.

Agustín Wilner es sociólogo por la Facultad de Ciencias Sociales de la Universidad de Buenos Aire (UBA). Es becario doctoral de Conicet, con sede en el Instituto de Investigaciones Gino Germani (UBA), donde también es integrante del equipo de investigación dirigido por la doctora Betina Freidin. Es docente de "Metodología y técnicas de la investigación social" de la carrera de Sociología de la UBA. Además, es estudiante de la maestría en Estudios Urbanos de la Universidad Nacional de General Sarmiento. Sus temas de interés son: las desigualdades sociales en salud, la segregación socioespacial y las desigualdades urbanas. ORCID: https://orcid.org/00000003-0006-2124

Entre sus publicaciones se encuentran:

Freidin, B., Wilner, A., Ballesteros. M. S. y Krause, M. (2018). Salud, riesgo ambiental y territorio: las experiencias de mujeres que viven en un barrio de clase popular en la periferia de Buenos Aires. Buenos Aires: Teseo.

Recepción: 26 de abril 2018.

Aceptación: 20 de julio de 2018. 
